\title{
Seasonal effect on fatigue, pain and dryness in primary Sjögren's syndrome
}

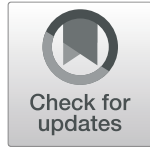

\author{
Pierre-Marie Duret ${ }^{1 *} \mathbb{D}$, Nicolas Meyer ${ }^{2}$, Alain Saraux ${ }^{3}$, Valérie Devauchelle-Pensec ${ }^{3}$, Raphaele Seror ${ }^{4}$, \\ Véronique Le-Guern ${ }^{5}$, Claire Larroche ${ }^{6}$, Aleth Perdriger ${ }^{7}$, Jean Sibilia ${ }^{8}$, Vianney Guardiolle ${ }^{2}$, Xavier Mariette ${ }^{4}$ \\ and Jacques-Eric Gottenberg ${ }^{8,9}$
}

\begin{abstract}
Background: To assess the presence of a seasonal effect on fatigue, pain and dryness in primary Sjögren's syndrome (pSS).

Methods: Data (date; visual analogue scales (VAS) for pain, fatigue and dryness) were extracted from three randomised placebo-controlled trials (RCTs) evaluating infliximab (TRIPSS; $n=103$ patients), hydroxychloroquine (JOQUER; $n=120$ patients) and rituximab (TEARS; $n=120$ patients) and from the 5 -year follow-up of the ASSESS prospective cohort ( $n=395$ patients). Data were analysed at each visit for each patient, according to the day, the month of the year and the season. Linear mixed models were used to take into account the repeated structure of the data and to analyse a potential cyclic effect.
\end{abstract}

Results: A total of 744, 584, 848 and 682 pain, fatigue and dryness VASs were collected on 632 subjects in spring, summer, fall and winter, respectively. No significant difference was observed in pain, fatigue and dryness, according to the month of the year or the season (all $p$ values $>0.05$ ).

Conclusion: In pSS, seasonality does not affect patient-reported outcomes (PROs) on fatigue, pain and dryness.

Keywords: Primary Sjögren's syndrome, Autoimmune diseases, Seasonality, Seasons and rheumatic diseases, Outcome measures, Patient-reported outcomes (PROs), Epidemiology, Clinical trials

\section{Background}

Seasonality in rheumatic diseases is an issue frequently perceived and voiced by patients. Several studies have identified weather-related flares in rheumatoid arthritis (RA) [1, 2]. Weather conditions might also influence pain and function in osteoarthritis [3], and a seasonal pattern in gout incidence has been described [4].

In addition, cyclic seasonal variations have been associated with disease onset and activity or worsening of symptoms in immune-mediated inflammatory diseases (IMIDs), in giant cell arteritis (GCA) [5], inflammatory myopathies [6], systemic lupus erythematosus (SLE) [7] and ANCA-associated vasculitis [8]. Interestingly, seasonal variations are not associated with clinical outcomes in psoriatic arthritis [9].

\footnotetext{
* Correspondence: pierre-marie.duret@ch-colmar.fr; pierremarie.duret@gmail.com

${ }^{1}$ Department of Rheumatology, Colmar General Hospital, Colmar, France Full list of author information is available at the end of the article
}

However, seasonality has not been investigated in primary Sjögren's syndrome (pSS) yet. Primary Sjögren's syndrome is the second most frequent systemic autoimmune disease, and it is clinically characterised by the disabling triad pain, dryness and fatigue and is immunologically associated with antinuclear antibodies, anti Ro/ SSA and/or La/SSB and the presence, in up to one third of the patients, of ectopic lymphoid structures developed in the target organs of the disease, which are mainly salivary and lacrimal glands [10].

Since fatigue, pain and dryness represent most of the disease burden shared by all patients with pSS, they compose the internationally validated ESSPRI (EULAR Sjögren's Syndrome Patient Reported Index) score [11], an outcome criteria evaluated in all on-going clinical trials.

Although fatigue, pain and dryness represent a major concern for patients with pSS, there is no data available

(c) The Author(s). 2020 Open Access This article is distributed under the terms of the Creative Commons Attribution 4.0 International License (http://creativecommons.org/licenses/by/4.0/), which permits unrestricted use, distribution, and 
in the literature regarding the potential variations of these symptoms according to seasonality.

Based on previous reports that have demonstrated, in $\mathrm{RA}$, a worsening of pain and fatigue in winter and an increase of dryness in spring in dry eye symptoms unrelated to pSS, it could be hypothesised that fall and winter are associated with increased fatigue and pain and spring and summer with exacerbation of ocular and oral dryness in pSS.

This study was therefore conducted to assess whether seasonal variations have an influence on pSS outcomes.

\section{Methods}

\section{Patient selection}

This study analysed patients from the French nationwide multicentre pSS cohort (Assessment of Systemic Signs and Evolution in Sjögren's Syndrome) ASSESS $(n=395)$ [12], established in 2006, with an available 5-year prospective follow-up and from three randomised placebocontrolled trials of infliximab (TRIPSS; $n=103$; followup of 22 weeks; 7 visits) [13], rituximab (TEARS; $n=$ 120; follow up of 24 weeks; 6 visits) [14] and hydroxychloroquine (JOQUER; $n=120$; follow up of 48 weeks; 4 visits) [15] (Fig. 1). All patients fulfilled the AmericanEuropean Consensus group criteria for pSS [16]. At each visit, in all studies, visual analogue scales (VASs) of patients for pain, fatigue and dryness were collected. Objective assessments of dryness (Schirmer test and unstimulated salivary flow) were collected in the ASSESS cohort at enrolment, second and fifth year of follow-up.

Since ASSESS is a non-interventional "natural history" cohort and all the three RCTs did not show any significant treatment effect, a combined analysis of all the available data was conducted.

\section{Statistical analysis}

Data combined from the four previous studies were aligned according to the days, months and seasons of the year. The dataset was collected over 10 years, from 2001 to 2002 (TRIPSS), 2008 to 2011 (TEARS), 2008 to 2012 (JOQUER) and from 2007 to 2014 in the ASSESS cohort.

Patients for whom one of the three VAS criteria (fatigue, pain or dryness) was missing were not included in the analysis.

Quantitative variables are described with the mean and its standard deviation (SD). Normality was assessed using the Shapiro-Wilk test.

Mean VAS for fatigue, pain and dryness were compared first across months and, secondly, in a separate model, across seasons using linear mixed models.

Given the availability, in the ASSESS cohort, of data concerning symptomatic treatments of dryness and systemic immunomodulatory treatments (Additional file 1:
Table S1), analyses were adjusted on two potential confounding factors which were age and treatment regimens assumed by patients. Linear mixed models were adjusted on symptomatic treatments of dryness (topical serum eye drops and pilocarpine hydrochloride) and systemic immunomodulatory treatments. The latter included hydroxychloroquine, corticosteroids, methotrexate, leflunomide, azathioprine, mycophenolate mofetil, cyclophosphamide (CYC) and rituximab (RTX) (CYC and RTX were considered as current treatment if prescribed in the 6 months prior to examination).

The models were fitted with a fixed time effect (month or season effect, in distinct models), with a random subject effect used to take into account the repeated structure of the data. In order to detect a cyclic seasonal effect, a cosinus transformation and a time shift was also applied to the time effect in separate models. Statistical analyses were performed using R3.3.1 with the LME4 and HGLM libraries. All statistical tests were two-sided and $p$ values $\leq 0.05$ were considered statistically significant.

\section{Results \\ Variations of fatigue pain and dryness intensity between seasons}

A total of 632 patients were analysed. We collected and analysed a total of 2858 VASs observations through the four studies (the number of patients withdrawn due to missing VAS data is shown on Fig. 1).

Table 1 shows the number of VASs for fatigue pain and dryness recorded in spring, summer, fall and winter and the variation of the ESSPRI score according to the season.

Mean (SD) pain VAS was 52.2 (27.9) on a 100-unit scale, 55.1 (28.1), 51.0 (28.7) and 51.7 (28.4) in spring, summer, fall and winter, respectively $(p=0.7541)$. Mean (SD) fatigue was 61.9 (23.2), 62.2 (25.2), 60.0 (25.5), and 61.9 (24.2), respectively $(p=0.7973)$. Mean (SD) dryness was 58.9 (21.8), 61.2 (22.9), 56.9 (22.8) and 57.9 (23.8), respectively $(p=0.4108)$. Moreover, the ESSPRI score, which is the mean of the three VASs values for a given patient, was 57.7 (24.3), 59.5 (24.5), 55.9 (25.7) and 57.2 (25.5) on a 100-unit scale, in spring, summer, fall and winter, respectively $(p=0.7288)$. None of these fluctuations was statistically significant $(p>0.05)$.

In addition, variations from month to month (Fig. 2a) or season to season (Table 1; Fig. 2b) of mean pain, fatigue and dryness were not significant either. Maximum between-months variation for pain was 7.2 on a 100-unit scale, 7.7/100 and 5.5/100 for dryness and fatigue, respectively. In addition, using cosine transform of time effect and different time lags, no time effect was observed in any model (data not shown). 


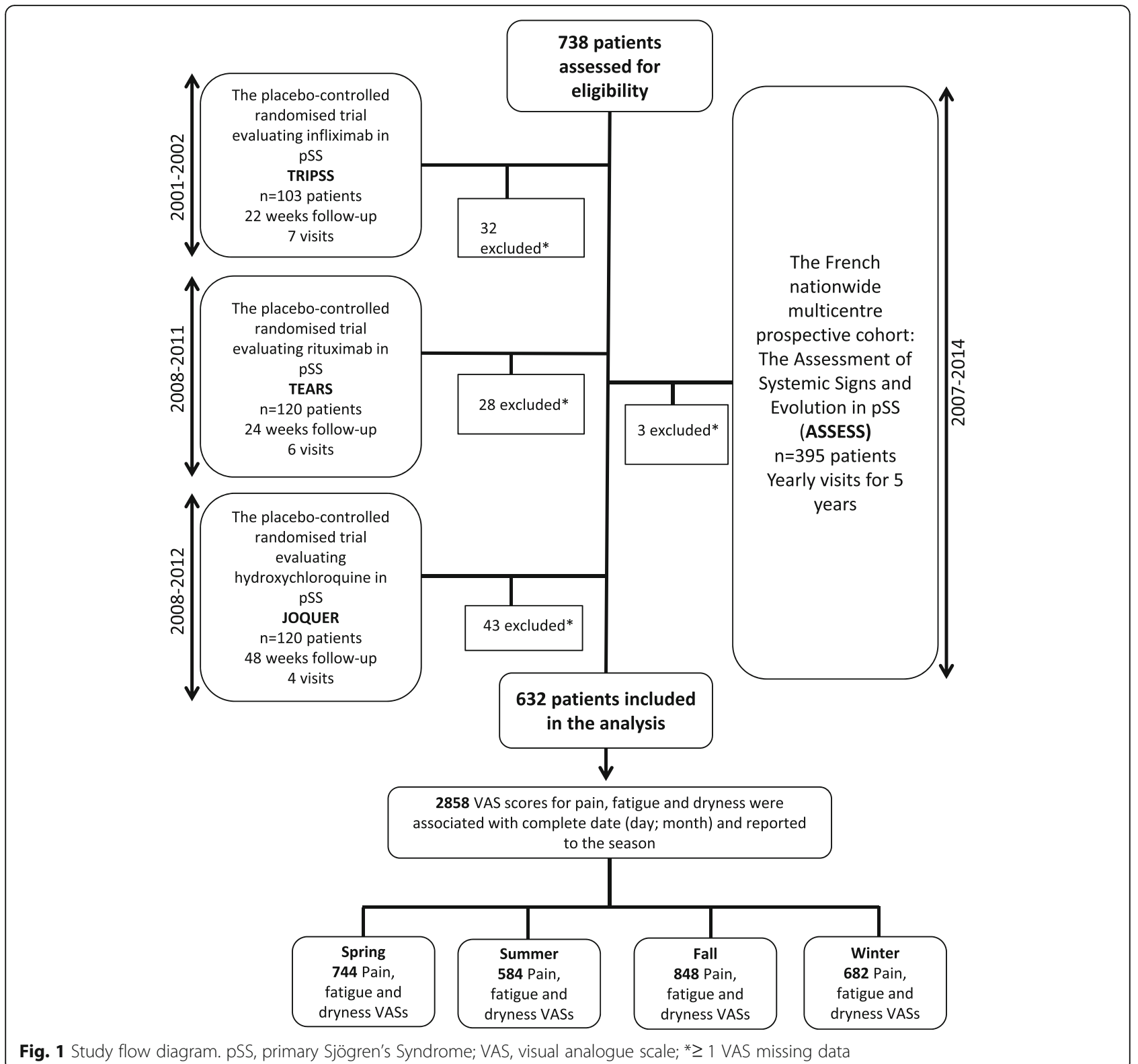

Table 1 Pain, fatigue and dryness by season for all spring, summer, fall and winter visits ( $n=2858$ observations) in 632 patients

\begin{tabular}{llllll}
\hline VAS & Spring & Summer & Fall & Winter & \multicolumn{1}{c}{$c$} \\
\hline$n^{*}$ & 744 & 584 & 848 & 582 \\
Pain $^{\#}$ mean (SD) & $52.2(27.9)$ & $55.1(28.1)$ & $51.0(28.7)$ & $51.7(28.4)$ & 0.7541 \\
Fatigue $^{\#}$ mean (SD) & $61.9(23.2)$ & $62.2(25.2)$ & $60.0(25.5)$ & $61.9(24.2)$ & 0.7973 \\
Dryness $^{\#}$ mean (SD) & $58.9(21.8)$ & $61.2(22.9)$ & $56.9(22.8)$ & $57.9(23.8)$ & 0.4108 \\
ESSPRI mean (SD) & $57.7(24.3)$ & $59.5(24.5)$ & $55.9(25.7)$ & $57.2(25.5)$ & 0.7288 \\
\hline
\end{tabular}

*Number of VAS; \#in mm; ESSPRI EULAR Sjögren's Syndrome Patient Reported Index 


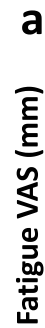
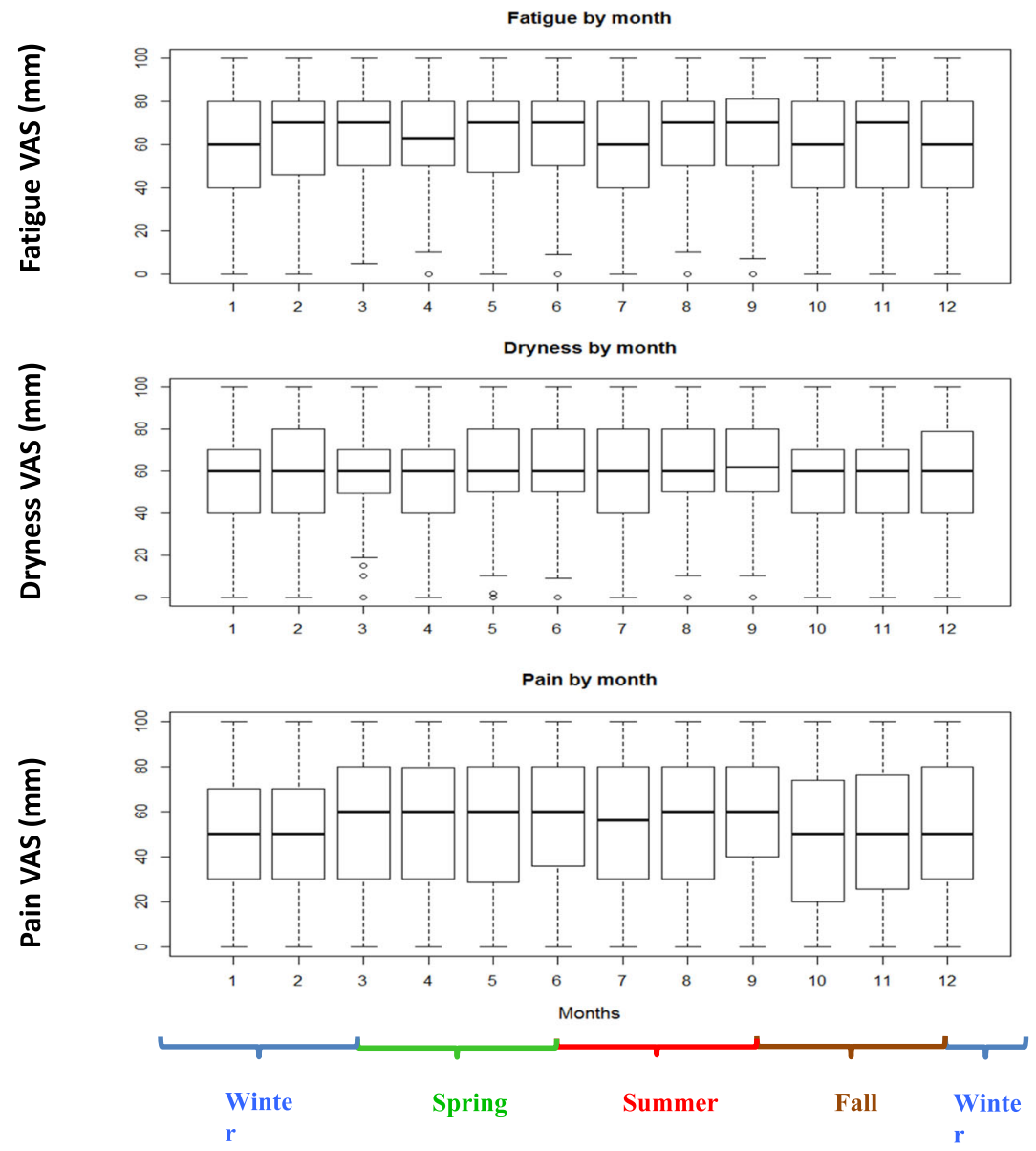

b
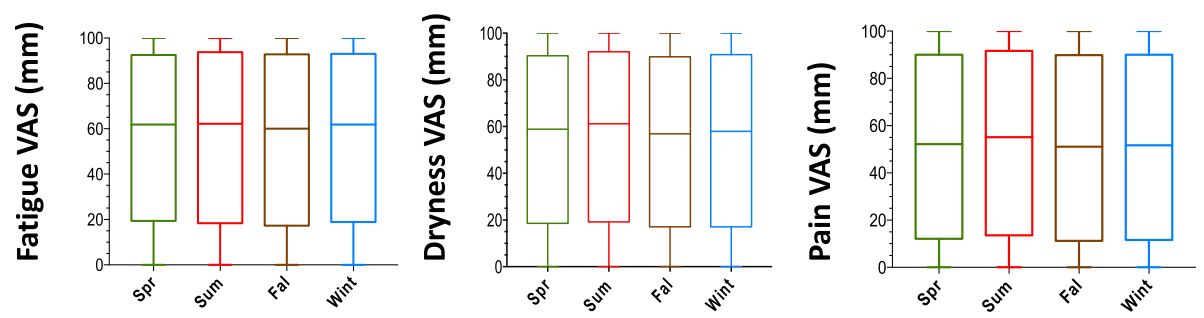

Fig. 2 Pain, fatigue and dryness fluctuations across months and seasons. a Box plots of VAS variations between months from January (month: 1) to December (month: 12). b Box plots of VAS variations between seasons. Spring (Spr) is depicted in green, summer (Sum) in red, fall (Fal) in brown and winter (Wint) in blue

Moreover, multivariate analyses were performed in the ASSESS cohort to address the influence on objective assessments of dryness (Schirmer test and unstimulated salivary flow) of potential confounding factors, such as age of patients, symptomatic treatments of dryness and systemic immunomodulatory treatments. Adjusted odds ratios (aOR) on age and treatment regimens did not detect any statistically significant seasonal effect on Schirmer test, with aOR of having a pathological Schirmer test $(\leq 5 \mathrm{~mm})$ of $0.93 \mathrm{CI} 95 \%$ [0.42-1.8] $(p=0.82)$,
$0.95 \mathrm{CI} 95 \%[0.43-2.14](p=0.89)$ and 0.75 CI 95\% $[0.38-1.53](p=0.35)$ in spring, summer and winter, respectively, in comparison to values collected in fall. Treatment regimens did not show any influence on salivary flow either, since aOR of having a pathological salivary flow (SF $<0.1 \mathrm{~mL} / \mathrm{min}$ ) was $1.23 \mathrm{CI} 95 \%$ [0.492.64] $(p=0.58), 0.71$ CI 95\% [0.24-1.43] $(p=0.38)$ and 0.83 CI 95\% [0.34-1.68] ( $p=0.6)$, in spring, summer and winter, respectively, in comparison to values collected in fall (Additional file 1: Table S2). 
The influence on PROs of age, symptomatic treatments of dryness and immunomodulatory treatments was also analysed in the ASSESS cohort. All the variations of pain, fatigue and dryness VASs and of the ESSPRI score over seasons, adjusted on age and treatment regimen were very limited and did not reach statistical significance. Adjusted pain VASs variations were 1.3 on a 100-unit scale, CI 95\% $[-1.6 ; 4.4](p=0.4), 0 / 100 \mathrm{CI} 95 \%[-3 ; 2.9](p=0.99)$ and $-0.3 / 100 \mathrm{CI} 95 \%[-3 ; 2.4](p=0.83)$, in spring, summer and winter, respectively, in comparison to values collected in fall. Adjusted fatigue VASs variations were 1.3/100 CI 95\% [-1.3; 4 ] $(p=0.33), 0.4 / 100[-2.2 ; 3](p=0.79)$, and $0.2 / 100[-2.7 ; 2.2](p=0.84)$ in spring, summer and winter, respectively, in comparison to values collected in fall. Adjusted dryness VASs variations were 1.3/100 CI 95\% [-1; 3.6] $(p=0.27), 2 / 100$ CI 95\% [- $0.3 ; 4.3](p=0.09)$ and $0.6 /$ 100 CI 95\% [-1.5; 2.8$](p=0.55)$, respectively, in comparison to values collected in fall. Finally, adjusted ESSPRI score changes were of $1.2 / 100 \mathrm{CI} 95 \%[-0.8 ; 3.2](p=0.25), 0.6 /$ 100 CI 95\% $[-1.4 ; 2.6](p=0.55)$ and $-0.2 / 100$ CI 95\% [$2.1 ; 1.6](p=0.82)$, respectively, in comparison to values collected in fall (Additional file 1: Table S3).

\section{Discussion}

The present study performed on a large population of patients and on a long duration did not show any seasonal effect on the main symptoms of pSS, pain, fatigue and dryness.

The pathogenesis of pSS is, not unlike other autoimmune diseases, considered multifactorial. A complex relationship between environmental and immunological factors affecting a peculiar genetic background may interact and sustain disease onset in susceptible individuals.

A seasonal pattern of several suspected environmental triggers, such as viral infections (EBV and CMV in winter and HBV and HCV in spring and summer) as well as sunshine exposure (through UV-B radiation, governing blood levels of inactive $25 \mathrm{OH}$-vitamin $\mathrm{D}_{3}$ ), might suggest an association between disease activity and season changes. Of note, previous studies have postulated that low levels of vitamin $\mathrm{D}$, because of its immunomodulatory effects, could affect clinical manifestations in patients with pSS [17], as demonstrated in other IMIDs, especially in LES [18] and RA [19]. Nevertheless, vitamin $D$ influence on pSS activity remains controversial.

Otherwise, weather-related ocular and oral dryness enhancement has been reported in patients outside autoimmune context, mainly in spring and summer, but this has never been specifically analysed in pSS.

Indeed, to our knowledge, there is no study available in the literature investigating a seasonal impact on symptoms and outcomes in primary Sjögren's syndrome.

In the present study, all the fluctuations observed were not statistically significant and were not clinically relevant either, since they were well lower than the minimal clinically important improvements (MCIIs) for dryness, pain and fatigue, which are $-10,-10$ and -20 on a 100-point scale, respectively [15].

Nevertheless, this study has several limitations. First, patients included in the ASSESS cohort underwent an annual evaluation of their symptoms and activity, mostly at the same period every year. However, the three trials lasted 1 year and visits were scattered all over the year. In addition, the statistical analysis not only focused on the intra-individual but also assessed the inter-individual seasonal variability of symptoms.

Several confounding factors, such as age, symptomatic treatments of dryness and immunomodulatory treatments assumed by patients, could have hampered the interpretation of the results. To address this point, linear mixed models were adjusted to take into account the influence of age and treatments on objective assessment of ocular and oral dryness and on PROs, in the ASSESS cohort. Adjusted analyses on age, immunomodulatory drug exposure and symptomatic treatments of dryness did not reveal any statistically significant effect of seasons on objective dryness outcomes. Furthermore, even when adjusted on age and treatments, there was no significant effect of seasons on fatigue, pain and dryness VASs or on the ESSPRI score either.

Other limitations include the unavailability of weather variables such as temperature, relative humidity, barometric pressure, sunshine exposure, precipitations and the variability of geographic locations (multicentric studies). A longitudinal study involving meteorological features and assessing pSS outcomes every months during several years, as recently performed in RA [20], could be the most accurate way to investigate the effect of seasonality in pSS.

\section{Conclusions}

This first large study on seasonality in pSS provides new evidence that fatigue, pain and dryness, as well as the ESSPRI score, do not have meaningful fluctuations according to months or seasons. In pSS, seasonality does not affect patient-reported outcomes (PROs) on fatigue, pain and dryness.

\section{Supplementary information}

Supplementary information accompanies this paper at https://doi.org/10. 1186/s13075-020-2118-1.

\footnotetext{
Additional file 1: Table S1. Drug regimens used at enrolment in the ASSESS cohort and number of patients with systemic immunomodulatory drugs and symptomatic treatments of dryness. Table S2. Influence of seasons on objective assessments of ocular and oral dryness (Schirmer test $\leq 5 \mathrm{~mm}$; Salivary flow $\leq 0.1 \mathrm{~mL} / \mathrm{min}$ ). Table S3. Variations of pain, fatigue and dryness VASs (on a 100-unit scale) and of the ESSPRI score according to seasons, adjusted on age, immunosuppressive treatments and symptomatic treatments of dryness.
} 


\section{Abbreviations}

ASSESS: Assessment of Systemic Signs and Evolution in Sjögren's Syndrome; Cl: Confidence interval; CMV: Cytomegalovirus; CYC: Cyclophosphamide; EBV: Epstein-Barr virus; ESSPRI: EULAR Sjögren's Syndrome Patient Reported Index; EULAR: European League Against Rheumatism; HBV: Hepatitis B virus; HCV: Hepatitis C virus; IMID: Immune-mediated inflammatory disease; IS: Immunosuppressive drugs; MCII: Minimal clinically important improvement; aOR: Adjusted odds ratio; PROs: Patient-reported outcomes; pSS: Primary Sjögren's Syndrome; RA: Rheumatoid arthritis; RCT: Randomised controlled trial; RTX: Rituximab; SF: Unstimulated salivary flow; SLE: Systemic lupus erythematosus; VAS: Visual analogue scale

\section{Acknowledgements}

The authors thank the patients with pSS included in the ASSESS cohort and patients who participated to TRIPSS, TEARS and JOQUER trials. ASSESS, TRIPSS and JOQUER were supported by the Assistance Publique des Hôpitaux de Paris (AP-HP) and TEARS by the Hôpital universitaire de la Cavale Blanche (Brest; France) with a grant from the French Ministry of Research (Programme Hospitalier de Recherche Clinique National; PHRC). The authors thank the French Society of Rheumatology for its grant to the ASSESS cohort.

\section{Authors' contributions}

All authors were involved in the drafting of the article or revising it critically for important intellectual content, and all authors approved the final version to be published. All authors were likewise involved in the study conception and design, acquisition of data and analysis and interpretation of data. PMD, NM, VG and JEG had full access to all the data in the study and will take responsibility for the integrity of the data and the accuracy of the data analysis.

\section{Funding}

The French Society of Rheumatology provided a grant to the cohort. The funder had no role in the study design, data collection and analysis, decision to publish or preparation of the manuscript.

\section{Availability of data and materials}

The datasets used and/or analysed during the current study are available from the corresponding author on reasonable request.

\section{Ethics approval and consent to participate}

The ASSESS cohort, promoted by the Assistance Publique des Hôpitaux de Paris, was approved by the Ethics Committee of Hôpital Bichat and the "Commission Nationale Informatique et Libertés". TEARS trial was reviewed and approved by the institutional review board of Hôpital universitaire de la Cavale Blanche (Brest; France); TRIPSS and JOQUER protocols were reviewed and approved by the institutional review board and Ethics Committee of Hôpital Bichat (Paris; France).

\section{Consent for publication}

Not applicable.

\section{Competing interests}

The authors declare that they have no competing interests.

\section{Author details}

'Department of Rheumatology, Colmar General Hospital, Colmar, France. 2Department of Public Health, GMRC, Strasbourg University Hospital, Strasbourg, France. ${ }^{3}$ Department of Rheumatology, Brest University Hospital, Brest, France. ${ }^{4}$ Center for Immunology of Viral Infections and Autoimmune Diseases, Assistance Publique - Hôpitaux de Paris, Hôpitaux Universitaires Paris-Sud, Le Kremlin-Bicêtre, INSERM, Paris, France. ${ }^{5}$ Department of Internal Medicine, Referral Center for Rare Autoimmune and Systemic Diseases, Hôpital Cochin, AP-HP, Université Paris Descartes, Paris, France. ${ }^{6}$ Department of internal Medicine, Avicenne Hospital, Bobigny, France. ${ }^{7}$ Department of Rheumatology, Rennes University Hospital, Rennes, France. ${ }^{8}$ Department of Rheumatology, Referral Center for Rare Autoimmune and Systemic Diseases, Strasbourg University Hospital, Strasbourg, France. ${ }^{9} \mathrm{CNRS}$, Immunopathologie et Chimie Thérapeutique/Laboratory of Excellence Medalis, Institut de Biologie Moléculaire et Cellulaire, Strasbourg, France.
Received: 6 July 2019 Accepted: 6 February 2020

Published online: 24 February 2020

\section{References}

1. Smedslund G, Mowinckel P, . Heiberg T, Kvien TK, Hagen KB. Does the weather really matter? A cohort study of influences of weather and solar conditions on daily variations of joint pain in patients with rheumatoid arthritis. Arthritis Care Res 2009;61(9):1243-1247.

2. likuni N, Nakajima A, Inoue E, Tanaka E, Okamoto H, Hara M, et al. What's in season for rheumatoid arthritis patients? Seasonal fluctuations in disease activity. Rheumatol Oxf Engl. 2007:46(5):846-8.

3. Dorleijn DMJ, Luijsterburg PAJ, Burdorf A, Rozendaal RM, Verhaar JAN, Bos PK, et al. Associations between weather conditions and clinical symptoms in patients with hip osteoarthritis: a 2-year cohort study. Pain. 2014;155(4):808-13.

4. Elliot AJ, Cross KW, Fleming DM. Seasonality and trends in the incidence and prevalence of gout in England and Wales 1994-2007. Ann Rheum Dis. 2009:68(11):1728-33.

5. Smeeth L, Cook C, Hall AJ. Incidence of diagnosed polymyalgia rheumatica and temporal arteritis in the United Kingdom, 1990-2001. Ann Rheum Dis. 2006;65(8):1093-8.

6. Leff RL, Burgess SH, Miller FW, Love LA, Targoff IN, Dalakas MC, et al. Distinct seasonal patterns in the onset of adult idiopathic inflammatory myopathy in patients with anti-Jo-1 and anti-signal recognition particle autoantibodies. Arthritis Rheum. 1991;34(11):1391-6.

7. Duarte-García A, Fang $\mathrm{H}$, To CH, Magder LS, Petri M. Seasonal variation in the activity of systemic lupus erythematosus. J Rheumatol. 2012;39(7):1392-8.

8. Kemna MJ, Cohen Tervaert JW, Broen K, Timmermans SAMEG, van Paassen $P$, Damoiseaux JGMC. Seasonal influence on the risk of relapse at a rise of antineutrophil cytoplasmic antibodies in vasculitis patients with renal involvement. J Rheumatol. 2017:44(4):473-81.

9. Touma Z, Eder L, Zisman D, Feld J, Chandran V, Rosen CF, et al. Seasonal variation in vitamin $\mathrm{D}$ levels in psoriatic arthritis patients from different latitudes and its association with clinical outcomes. Arthritis Care Res. 2011;63(10):1440-7.

10. Mariette X, Criswell LA. Primary Sjögren's syndrome. N Engl J Med. 2018;379(1):97.

11. Seror R, Ravaud P, Mariette X, Bootsma H, Theander E, Hansen A, et al. EULAR Sjogren's Syndrome Patient Reported Index (ESSPRI): development of a consensus patient index for primary Sjogren's syndrome. Ann Rheum Dis. 2011;70(6):968-72.

12. Gottenberg J-E, Seror R, Miceli-Richard C, Benessiano J, Devauchelle-Pensec $V$, Dieude P, et al. Serum levels of beta2-microglobulin and free light chains of immunoglobulins are associated with systemic disease activity in primary Sjögren's syndrome. Data at Enrollment in the Prospective ASSESS Cohort. Plos One. 2013;8(5):e59868.

13. Mariette X, Ravaud P, Steinfeld S, Baron G, Goetz J, Hachulla E, et al. Inefficacy of infliximab in primary Sjögren's syndrome: results of the randomized, controlled Trial of Remicade in Primary Sjögren's Syndrome (TRIPSS). Arthritis Rheum. 2004;50(4):1270-6.

14. Devauchelle-Pensec V, Mariette X, Jousse-Joulin S, Berthelot J-M, Perdriger A, Puéchal $X$, et al. Treatment of primary Sjögren syndrome with rituximab: a randomized trial. Ann Intern Med. 2014;160(4):233-42.

15. Gottenberg J-E, Ravaud P, Puéchal X, Le Guern V, Sibilia J, Goeb V, et al. Effects of hydroxychloroquine on symptomatic improvement in primary Sjögren syndrome: the JOQUER randomized clinical trial. JAMA. 2014;312(3):249-58.

16. Vitali C, Bombardieri S, Jonsson R, Moutsopoulos HM, Alexander EL, Carsons $S E$, et al. Classification criteria for Sjögren's syndrome: a revised version of the European criteria proposed by the American-European Consensus Group. Ann Rheum Dis. 2002;61 (6):554-8.

17. Bang B, Asmussen K, Sørensen OH, Oxholm P. Reduced 25-hydroxyvitamin $\mathrm{D}$ levels in primary Sjögren's syndrome. Correlations to disease manifestations. Scand J Rheumatol. 1999;28(3):180-3.

18. Cutolo M, Otsa K. Review: vitamin D, immunity and lupus. Lupus. 2008;17(1):6-10.

19. Cutolo M, Otsa K, Laas K, Yprus M, Lehtme R, Secchi ME, et al. Circannual vitamin $\mathrm{d}$ serum levels and disease activity in rheumatoid arthritis: Northern versus Southern Europe. Clin Exp Rheumatol. 2006;24(6):702-4.

20. Mandl P, Alasti F, Kaltenberger R, et al. FRl0092 Influence of Temperature and Humidity on Disease Activity in Rheumatoid Arthritis. Ann Rheum Dis 2016;75:460.3-460

\section{Publisher's Note}

Springer Nature remains neutral with regard to jurisdictional claims in published maps and institutional affiliations. 